\title{
Correction to: A complete classification of 5-regular circulant graphs that allow cyclic orthogonal double covers
}

\author{
M. Higazy ${ }^{1,2}\left(D \cdot\right.$ R. Scapellato ${ }^{3} \cdot$ Y. S. Hamed ${ }^{1,2}$
}

Published online: 2 March 2021

(๑) Springer Science+Business Media, LLC, part of Springer Nature 2021

\section{Correction to: J Algebr Comb}

\section{https://doi.org/10.1007/s10801-020-01008-4}

The original version of the article contained errors in citation.

The citation errors are given below:

Page 2, line 11: [2] must be [1].

Page 2, line 15: [3] must be [2].

Page 2, line 19: [4] must be [3].

Page 2, line -13: [3] must be [2].

Page 2, line -7: [5, 6] must be $[4,5]$.

Page 3, line 5: [6] must be [5].

Page 3, line 6: [3] must be [2].

Page 3, line 7: [6] must be [5].

Page 3, line 10: [7] must be [6].

Page 3, line 11: [8] must be [7].

Page 3, line 12: [7] must be [6].

Page 3, line 13: [8] must be [7].

Page 3, line 14: [6] must be [5].

The original article can be found online at https://doi.org/10.1007/s10801-020-01008-4.

$\bowtie$ M. Higazy

m.higazy@tu.edu.sa

R. Scapellato

raffaele.scapellato@polimi.it

Y. S. Hamed

yasersalah@tu.edu.sa; king_raphael@yahoo.it

1 Department of Mathematics and Statistics, College of Science, Taif University, Taif 21974, Saudi Arabia

2 Department of Physics and Engineering Mathematics, Faculty of Electronic Engineering, Menoufia University, Menouf 32952, Egypt

3 Dipartimento di Matematica, Politecnico di Milano, Piazza Leonardo da Vinci 32, 20133 Milan, Italy 
Page 3, line 16: $[9,10]]$ must be $[8,9]$.

Page 3, line 16: [11] must be [10].

The original article has been corrected.

Publisher's Note Springer Nature remains neutral with regard to jurisdictional claims in published maps and institutional affiliations. 